\title{
Monitoring and assessment of urban growth patterns using spatio-temporal built-up area analysis
}

\begin{abstract}
The identification of spatio-temporal patterns of the urban growth phenomenon has become one of the most significant challenges in monitoring and assessing current and future trends of the urban growth issue. Therefore, spatio-temporal and quantitative techniques should be used hand in hand for a deeper understanding of various aspects of urban growth. The main purpose of this study is to monitor and assess the significant patterns of urban growth in Seremban using a spatio-temporal built-up area analysis. The concentric circles approach was used to measure the compactness and dispersion of built-up area by employing Shannon's Entropy method. The spatial directions approach was also utilised to measure the sustainability and speed of development, while the gradient approach was used to measure urban dynamics by employing landscape matrices. The overall results confirm that urban growth in Seremban is dispersed, unbalanced and unsustainable with a rapid speed of regional development. The main contribution of using existing methods with other methods is to provide several spatial and statistical dimensions that can help researchers, decision makers and local authorities understand the trend of growth and its patterns in order to take the appropriate decisions for future urban planning. For example, Shannon's Entropy findings indicate a high value of dispersion between the years 1990 and 2000 and from 2010 to 2016 with a growth rate of approximately 94 and 14\%, respectively. Therefore, these results can help and support decision makers to implement alternative urban forms such as the compactness form to achieve an urban form that is more suitable and sustainable. The results of this study confirm the importance of using spatio-temporal built-up area and quantitative analysis to protect the sustainability of land use, as well as to improve the urban planning system via the effective monitoring and assessment of urban growth trends and patterns.
\end{abstract}

Keyword: Urban growth; GIS; Quantitative analysis; Built-up area analysis; Monitoring 